\title{
The impact of future space observatories on planetary nebula research
}

\author{
Michael J. Barlow \\ Dept. of Physics \& Astronomy, University College London, Gower Street, London WC1E 6BT
}

\begin{abstract}
A comparison between the past four decades of astrophysical space missions and those expected to be launched over the next decade shows a marked decrease in numbers. However, missions such as Gaia and the JWST are expected to have a major impact on planetary nebula research. The capabilities of these and other anticipated space missions are discussed.
\end{abstract}

Keywords. planetary nebulae: general, space observatories

\section{Introduction}

Like many other astrophysical fields, from the 1970's onwards planetary nebula research has benefited greatly from the availability of powerful space observatories operating at wavelengths from the X-ray to the infrared. Table 1 summarises, decade by decade, the principal past, current and planned general astrophysics space missions.

What are the prospects for the space observatories listed in Table 1 that are currently still fully operational? ESA's Herschel Space Observatory was launched in 2009 and current projections point to complete helium boil-off, and therefore the end of the mission, occurring in late-2012 or early-2013. For the Hubble Space Telescope the main components at risk are believed to be its complement of detectors, with current estimates suggesting a $50 \%$ probability by $2015 / 6$ of catastrophic failure due to cumulative radiation damage.

At X-ray wavelengths, prospects for the current two observatories operating in space are mixed. NASA's Chandra observatory, launched in 1999, is expected to be able to stay operational until 2015/6, while the expectations for ESA's XMM-Newton observatory, also launched in 1999, are better - a recent review has concluded that it could remain operational till 2025.

While six to nine new observatories have been launched in each of the four decades since 1971, the prospects for the current decade appear bleaker at present, with only four new general astrophysics missions, GEMS, Astrosat, Gaia and the James Webb Space Telescope (JWST), expected to have been launched by 2020.

Currently planned for launch in 2014, GEMS, the Gravity and Extreme Magnetism Small Explorer, will measure X-ray polarization in order to study spinning black holes and probe magnetic field around neutron stars. The other three missions, Astrosat, Gaia and the JWST, are discussed in more detail below.

\section{The Astrosat Mission}

Astrosat is an Indian Space Research Organisation mission for X-ray and UV/optical astronomy, scheduled for launch in 2012 and expected to have a lifetime in excess of 5 years. In addition to having four different X-ray instruments (see Table 2), it will also be equipped with two 40-cm telescopes for UV and optical imaging, each with an angular resolution of 1.8 arcsec and capable of measuring 20 th magnitude objects in 1800 s. There 
Table 1. Past and Future Space Missions for General Astrophysics ${ }^{1}$

\begin{tabular}{|c|c|c|c|c|}
\hline Decade & X-ray & UV or Optical & Infrared & TOTAL \\
\hline 1971-1980 & $\begin{array}{l}4 \\
\text { Ariel-5, SAS-3, } \\
\text { HEAO-1, Einstein }\end{array}$ & $\begin{array}{l}4 \\
\text { TD-1, Copernicus, } \\
\text { ANS, IUE }\end{array}$ & 1 AFGL & 9 \\
\hline 1981-1990 & $\begin{array}{l}3 \\
\text { Exosat, Ginga, ROSAT }\end{array}$ & $\begin{array}{l}2 \\
\text { Astron, HST }\end{array}$ & $\begin{array}{l}1 \\
\text { IRAS }\end{array}$ & 6 \\
\hline 1991-2000 & $\begin{array}{l}4 \\
\text { ASCA, RXTE, Chandra } \\
\text { XMM-Newton }\end{array}$ & $\begin{array}{l}3 \\
\text { EUVE, FUSE } \\
\text { Hipparcos }\end{array}$ & $\stackrel{2}{\text { ISO, }}$ MSX & 9 \\
\hline 2001-2010 & $\begin{array}{l}1 \\
\text { Suzaku }\end{array}$ & 1 & $\begin{array}{l}4 \\
\text { Spitzer, AKARI, } \\
\text { Herschel, WISE }\end{array}$ & 6 \\
\hline 2011-2020 & $\begin{array}{l}2 \\
\text { GEMS, Astrosat } \\
\sim 2014,2012\end{array}$ & $\begin{array}{l}2 \\
\text { Astrosat, GAIA } \\
2012,2013\end{array}$ & $\begin{array}{l}(1) \\
\text { JWST } \\
2018 ?\end{array}$ & $(5)$ \\
\hline TOTAL & 14 & 12 & (9) & 35 \\
\hline Post-2020 & ATHENA? & WSO? & SPICA? & \\
\hline
\end{tabular}

${ }^{1}$ Missions with particular impact on planetary nebula research are boldfaced.

Table 2. Astrosat X-ray and UV/Optical Science Instruments

\begin{tabular}{lllll}
\hline \hline Instrument & Energy Range & Description & Ang. Res. & Sensitivity \\
& & & \\
\hline & & & & \\
SXT & $0.3-8 \mathrm{keV}$ & FOV $=42^{\prime}$ & $3-4 \operatorname{arcmin}$ & $0.01 \mathrm{mCrab}$ in $10^{4} \mathrm{~s}$ \\
LAXPC & $2-80 \mathrm{keV}$ & 3 Prop. Counters & $5 \operatorname{arcmin}$ & $0.1 \mathrm{mCrab}$ in $10^{3} \mathrm{~s}$ \\
CZT & $10-150 \mathrm{keV}$ & Hard X-ray Imager & 8 arcmin & $0.5 \mathrm{mCrab}$ in $10^{3} \mathrm{~s}$ \\
SSM & $2-10 \mathrm{keV}$ & Scanning Sky Monitor & $5-10 \operatorname{arcmin}$ & $50 \mathrm{mCrab}$ in $300 \mathrm{~s}$ \\
UVIT & $1300-5500 \AA$ & Twin 40-cm Telescopes & $1.8 \operatorname{arcsec}$ & $21 \mathrm{st}$ mag in $1800 \mathrm{~s}$ \\
\hline
\end{tabular}

will be one broad-band far-UV filter (1300-1500 A), four broad-band and two narrowband near-UV filters, plus four broad-band filters in the 3000-5500- $\AA$ region. Further details are available at www.iucaa.ernet.in/ astrosat/. Given the very low number of space missions currently planned for the X-ray and UV/optical domains, Astrosat is a welcome additional resource, with potential interest for studies of PN central stars.

\section{Gaia}

Gaia is an ambitious ESA mission which aims to measure parallaxes and proper motions for one billion stars out to distances of $50 \mathrm{kpc}$. During its L2 five-year mission, beginning in 2013, it will survey the whole sky in order to achieve astrometric completeness down to $\mathrm{V}=20$. Its gigapixel imaging system consists of $106 \mathrm{CCD}$ sensors each with $1966 \times 4500$ 
pixels. Final positional accuracies will be 7, 20 and $300 \mu \operatorname{arcsec}$ at $\mathrm{V}=10,15$ and 20, respectively. Distances accurate to $1 \%$ are expected to be obtained for $10^{7}$ stars out to $2.5 \mathrm{kpc}$, as well as distances accurate to $10 \%$ for $10^{8}$ stars out to $25 \mathrm{kpc}$. Many PN central stars will therefore have accurate distances available by the end of the Gaia mission. The most favourable cases will be for central stars in low surface brightness nebulae, i.e. old $\mathrm{PNe}$, whereas central stars in higher surface brightness PNe will be more problematic (see below).

Gaia has twin telescopes, each with a with rectangular $1.49 \times 0.54 \mathrm{~m}$ primary, with a fixed wide angle between their lines of sight (LOS). The mirrors in each telescope system are made of sintered silicon carbide, with enhanced silver reflective coatings to optimise their reflectivities in the $3200-10000 \AA$ region. The spacecraft will rotate around an axis perpendicular to the LOS's of the two telescopes, with a rigid frame of reference being obtained by measuring the relative positions of objects from both observing directions. Each object will be measured about 70 times during the 5-year mission.

Gaia will have three instruments: (1) an astrometric instrument dedicated to measuring the angular positions of stars with V magnitudes in the range 6-20; (2) a photometric instrument covering the same magnitude range, which will acquire spectrophotometry at ultralow spectral resolution and will subsequently convolve the spectra with a range of filter pass-band profiles to generate photometry in 4 broad bands and 11 medium bands, including an $\mathrm{H} \alpha$ band; (c) a high-resolution spectrometer covering the 8470-8740 A region at $\mathrm{R}=11,500$, which will use the Ca II infrared triplet lines and the $\mathrm{H}$ I Paschen-14 line to measure stellar radial velocities to an accuracy of better than $15 \mathrm{~km} \mathrm{~s}^{-1}$ down to $\mathrm{V}=17$.

Observations from the photometric instrument should be of particular interest to planetary nebula research. As Gaia rotates across the sky, the spectrophotometer splits the incoming light and sends it through prisms to blue- and red-sensitive CCDs, which will respectively cover the $3300-6600 \AA$ and $6500-10500 \AA$ wavelength ranges. The resulting spectra will have a dispersion which is a function of wavelength, ranging from 40$320 \AA$ pixel $^{-1}$ in the blue and $70-150 \AA$ pixel $^{-1}$ in the red. These spectra have the potential to discover a significant number of young compact PNe and post-AGB objects with emission lines. However, current plans are that data on objects found to have angular diameters larger than 0.7 arcsec will be thrown away, i.e. not transmitted to the ground, which will clearly impact on prospects for measurements of PNe with medium to high nebular surface brightnesses.

\section{The James Webb Space Telescope}

As is well known, the JWST is aimed to be NASA's flagship successor to the Hubble Space Telescope, with a $15 \%$ funding contribution from ESA. It is currently beset by political problems in the US, with a House sub-committee attempting in July 2011 to axe the project, after $\sim \$ 4$ bn had already been spent. However, the equivalent Senate sub-committee restored funding for the JWST in their version of the spending bill and it appears that it may survive in an eventual reconciled bill. However, budgetary issues had already forced a delay in its expected launch date, from 2014 to 2018.

The JWST will have a deployable segmented-mirror primary, equivalent in collecting area to a single mirror with a $6.55 \mathrm{~m}$ diameter, which will use passive cooling to achieve an operating temperature of $\sim 45 \mathrm{~K}$ at its L2 station, versus $\sim 80 \mathrm{~K}$ for the 3.5 -m mirror of the Herschel Space Observatory. The JWST will have four instruments, a 0.6-5 $\mu \mathrm{m}$ near-IR wide field camera (NIRCam), which will have a coronagraphic mask available; a 1-5 $\mu \mathrm{m}$ near-IR multi-object spectrometer (NIRSpec); a 5-18 $\mu \mathrm{m}$ mid-IR camera and spectrometer (MIRI), also with a coronagraphic mask available; and a Tunable Filter 
Table 3. JWST Instrument Properties

\begin{tabular}{|c|c|c|c|c|c|}
\hline Instrument & $\begin{array}{l}\lambda \text {-range } \\
(\mu \mathrm{m})\end{array}$ & Features & Detectors & $\begin{array}{l}\text { Platescale } \\
\text { (arcsec) }\end{array}$ & FoV \\
\hline NIRCam (SW) & $0.6-2.3$ & $\begin{array}{l}\mathrm{R} \sim 4,10,100 \text { filters } \\
+ \text { coronagraph }\end{array}$ & $\begin{array}{l}\text { Two } 2 \times 2 \text { mosaics } \\
\text { of } 2048 \times 2048\end{array}$ & 32 & $2.2 \times 4.4$ \\
\hline NIRCam (LW) & $2.4-5.0$ & $\begin{array}{l}\mathrm{R} \sim 4,10,100 \text { filters } \\
\text { + coronagraph }\end{array}$ & $\begin{array}{l}\text { Two } 2 \times 2 \text { mosaics } \\
\text { of } 2048 \times 2048\end{array}$ & 65 & $2.2 \times 4.4$ \\
\hline NIRSpec $R=100$ & $0.6-5.0$ & Slits/microshutters & Two $2048 \times 2048$ & 100 & $3.4 \times 3.1$ \\
\hline NIRSpec $R=1000$ & $1.0-5.0$ & Slits/microshutters & Two $2048 \times 2048$ & 100 & $3.4 \times 3.1$ \\
\hline NIRSpec $R=3000$ & $1.0-5.0$ & Integral Field Unit & Two $2048 \times 2048$ & 100 & $3.0 \times 3.0$ \\
\hline MIRI (Imaging) & $5-28$ & Filters, coronagraph & $1024 \times 1024$ & 110 & $1.4 \times 1.9$ \\
\hline MIRI (Prism) & $5-10$ & $\mathrm{R}=100$ & $1024 \times 1024$ & 110 & $1.4 \times 1.9$ \\
\hline MIRI (Echelle) & $5-28$ & IFU $R=3000$ & Two $1024 \times 1024$ & $220-470$ & $3.6-7.5 "$ \\
\hline FGS-TFI (SW) & $1.5-2.5$ & $\mathrm{R} \sim 100$ etalon & $2048 \times 2048$ & 68 & $2.3 \times 2.3$ \\
\hline FGS-TFI (LW) & $3.2-5.0$ & $\mathrm{R} \sim 100$ etalon & $2048 \times 2048$ & 68 & $2.3 \times 2.3$ \\
\hline
\end{tabular}

Imager (TFI), associated with the optical bench of the Fine Guidance Sensor (FGS) and covering the 1.5-2.5 and 3.2-5.0 $\mu \mathrm{m}$ regions with a resolving power of $75-120$. Table 3 summarises the main properties of the four science instruments on the JWST, while Fig. 1 compares the expected point source sensitivities of the NIRCam and MIRI imagers with those of several other spaceborne, airborne and ground-based telescopes. At mid-IR wavelengths Spitzer went nearly a factor of 100 deeper than ground-based 8-m telescopes, while JWST will go a factor of 50 deeper than Spitzer. Since young PNe and post-AGB objects usually show strong dust continuum, dust feature and line emission in the 10-20$\mu \mathrm{m}$ region, they should be easily detectable out to very large distances with the $J W S T$.

JWST's huge sensitivity gains have one important consequence, however, namely that its saturation limits will be at unprecedently faint levels, preventing observations of many sources in the Milky Way and Magellanic Clouds. For example, even MIRI's R=3000 spectroscopic mode will saturate on sources with $10-\mu \mathrm{m}$ fluxes of only $\sim 0.5 \mathrm{Jy}$. The LMC post-AGB object SMP 11, which shows a rich mid-IR absorption spectrum from organic molecules such as benzene (Bernard-Salas et al. 2006), has a 10- $\mu$ m flux only just below this level. Photometric studies of AGB stars, post-AGB objects and PNe using NIRCam and MIRI will often only be feasible for targets lying at greater distances than the Magellanic Clouds.

LMC SMP 11 would be detectable at $10 \sigma$ in $10^{4} \mathrm{sec}$ out to a distance of $18 \mathrm{Mpc}$ at $18 \mu \mathrm{m}$; out to a distance of $38 \mathrm{Mpc}$ at $10 \mu \mathrm{m}$; and out to a distance of $47 \mathrm{Mpc}$ at $7.5 \mu \mathrm{m}$. Objects discovered via imaging photometry could be followed up from 5-10 $\mu \mathrm{m}$ with MIRI's long-slit $\mathrm{R}=100$ spectrometer. SMP LMC11 would yield $10 \sigma$ per spectral resolution element in $10^{4} \mathrm{sec}$ at a distance of $14.5 \mathrm{Mpc}$. These numbers indicate that in the above modes MIRI should be comfortably capable of studying similar objects out to the Virgo Cluster $(\mathrm{D} \sim 14 \mathrm{Mpc}$ ) and beyond.

Planetary nebulae have become important probes of extragalactic stellar systems, using the bright [O III] $5007 \AA$ line, in which up to $10 \%$ of the nebular luminosity can be emitted. Arnaboldi et al. (1996) measured velocities for 19 PNe in the outer regions of the giant elliptical galaxy NGC 4406, in the southern Virgo extension region. Although 
Photometric performance, point source

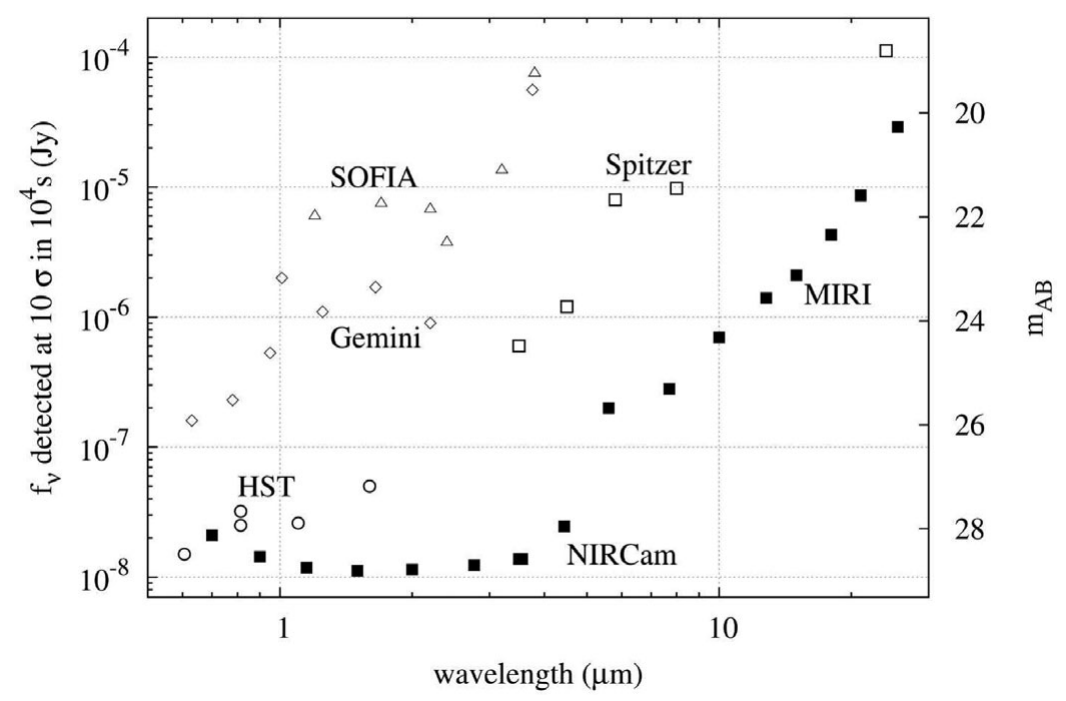

Figure 1. $10^{4} \mathrm{~s} 10-\sigma$ point source detection limits for JWST's NIRCam and MIRI instruments, compared to those for HST, Spitzer, SOFIA and 8-m Gemini instruments, illustrating the very large sensitivity gains anticipated.
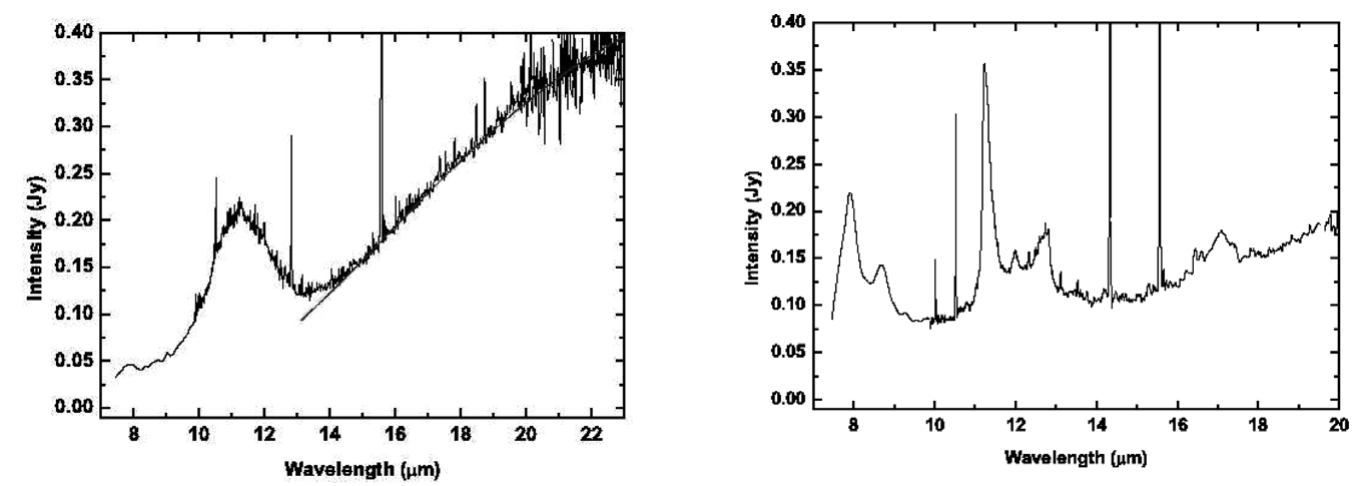

Figure 2. Spitzer-IRS hi-res spectra of (left) SMP LMC 8, showing a strong SiC 11.2- $\mu \mathrm{m}$ emission feature and (right) SMP LMC 36, showing very strong PAH emission bands. Both planetary nebulae also exhibit narrow fine structure ionic emission lines in their spectra. The IRS lo-res spectra of these PNe are shown by Bernard-Salas et al. (2009)

this galaxy has a peculiar radial velocity of $-227 \mathrm{~km} \mathrm{~s}^{-1}$, three of the PNe had velocities close to $1400 \mathrm{~km} \mathrm{~s}^{-1}$, the mean radial velocity of the Virgo cluster. It was concluded that they were intracluster PNe. Theuns \& Warren (1997) discovered ten PN candidates in the Fornax cluster, in fields well away from any Fornax galaxy - consistent with tidal stripping of cluster galaxies. They estimated that intracluster stars could account for up to $40 \%$ of all the stars in the Fornax cluster. Méndez et al. (1997) surveyed a $50 \operatorname{arcmin}^{2}$ area near the centre of the Virgo cluster, detecting 11 PN candidates. From this, they estimated a total stellar mass of about $4 \times 10^{9} \mathrm{M}_{\odot}$ in their survey area and that such a population could account for up to $50 \%$ of the total stellar mass in the Virgo cluster.

The luminous intergalactic PNe discovered to date at optical wavelengths therefore represent the tip of a very large iceberg. Infrared observations with JWST of dust-emitting 


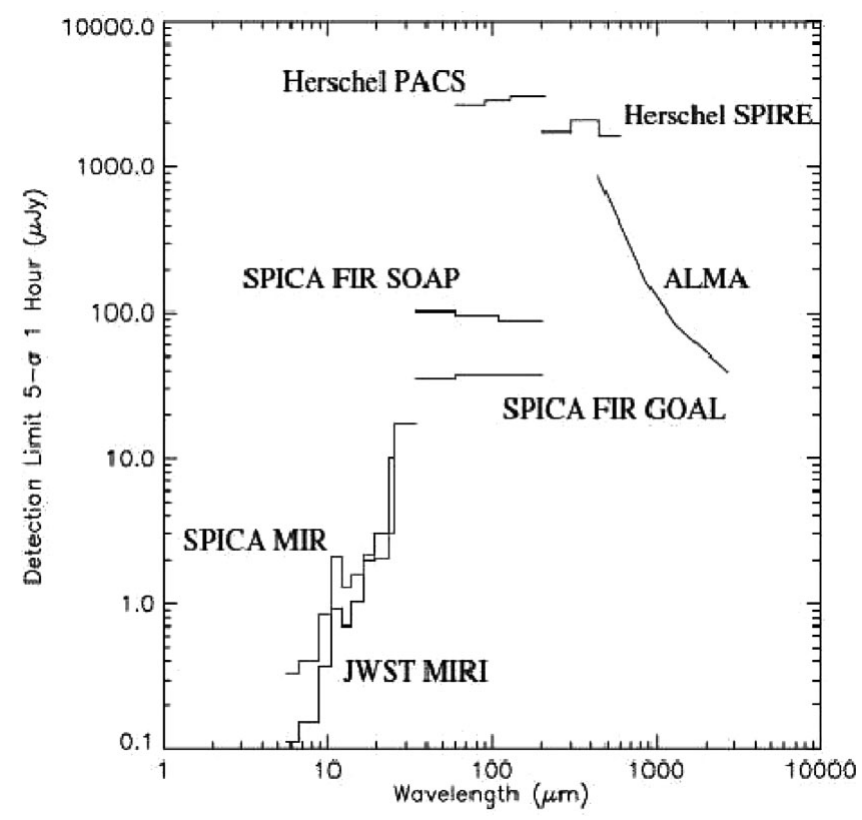

Figure 3. Predicted point source photometric detection sensitivities, in $\mu \mathrm{Jy}$ (5- $\sigma, 1$-hour), for SPICA, compared to those for the JWST, Herschel and ALMA. From Swinyard \& Nakagawa (2009).

AGB stars and planetary nebulae located in intergalactic regions of the Virgo Cluster appear feasible. Figure 2 shows the Spitzer mid-infrared spectra of two LMC planetary nebulae. The fluxes measured for the SiC-emitting nebula SMP LMC 8 indicate that in $10^{4} \mathrm{sec}$ MIRI imaging would yield $10 \sigma$ detections for a distance of $\mathrm{D}=19 \mathrm{Mpc}$ with the 7.7 - and $11.3-\mu \mathrm{m}$ filters and for a distance of $13 \mathrm{Mpc}$ with the $18-\mu \mathrm{m}$ filter. For the strongly PAH-emitting PN SMP LMC 36 (Fig. 2), in $10^{4}$ sec MIRI imaging would yield a $10 \sigma$ detection for a distance of $44 \mathrm{Mpc}$ with the 7.7- $\mu \mathrm{m}$ filter and for a distance of $23 \mathrm{Mpc}$ with the 11.3- $\mu \mathrm{m}$ filter. The carbon star MSX SMC 159 (Sloan et al. 2006), would yield $10 \sigma$ detections in $10^{4} \mathrm{sec}$ with MIRI out to a distance of $\mathrm{D}=29 \mathrm{Mpc}$ with the $5.6-\mu \mathrm{m}$ filter, to $23 \mathrm{Mpc}$ with the $7.7-\mu \mathrm{m}$ filter and to $12 \mathrm{Mpc}$ with the $11.3-\mu \mathrm{m}$ filter. Similar detection limits apply for oxygen-rich AGB stars. As shown by Groenewegen et al. (2007), mid-infrared colour indices can be used to estimate mass loss rates and thereby mass inputs from stellar populations hosting AGB stars.

The other JWST instruments will also be useful for such studies, e.g. with the Tunable Filter Imager $(\mathrm{R}=100)$ a carbon star similar to MSX SMC 159 would give $10 \sigma$ per resolution element in $10^{4} \mathrm{sec}$ for distances out to $\mathrm{D}=24-29 \mathrm{Mpc}$, for wavelengths from $3 \mu \mathrm{m}$. There are at least 20-30 AGB stars for every PN, so there should be many detectable AGB stars in Virgo Cluster fields, allowing the total stellar population to be determined, as well as their gas and dust mass inputs into the intracluster medium.

\section{Other Future Missions}

SPICA is a Japanese-led infrared mission, with ESA participation, which is currently at an advanced design stage, awaiting Phase B approval. It will cover the 5 -210- $\mu \mathrm{m}$ wavelength range with a $3.5-\mathrm{m}$ primary cooled to less than $5 \mathrm{~K}$ by mechanical cryocoolers, giving it a similar mid-IR sensitivity to that of the $J W S T$, despite its smaller primary, and 
a far-IR sensitivity a factor of 100 better than Herschel's, see Figure 3. To be launched to an L2 station by the end of the decade, it has a mission lifetime goal of 5 years, potentially extendable to 10 years. It will be equipped with a mid-IR imaging spectrophotometer and coronagraph, a mid-IR medium and high resolution spectrometer and a far-IR imaging spectrometer. As well as supplying the telescope, ESA would provide the far-IR imaging spectrometer, called SAFARI. SPICA's wide-field mid-IR and far-IR cameras and imaging spectrometers will give it a powerful capability to detect IR continuum- and line-emitting objects in blind surveys

The 2.4-m HST's STIS and COS spectrometers currently provide the sole ultraviolet spectroscopic capability to astronomers. However, UV spectroscopic observations receive only a minority of the total time allocated on the the HST and beyond 2015/6 Hubble's capabilities may start to fail. There is a clear need for a dedicated UV spectroscopic mission. The 'World Space Observatory' (WSO) is based on a concept that was pioneered and vigorously pursued by the late Willem Wamsteker and is currently a Russian-led international collaboration, see http://wso.inasan.ru/. Its 1.7-m telescope would be equipped with $\mathrm{R}=50,000$ spectrometers (Germany) covering the $1020-3100$ - $\AA$ region, a long-slit spectrometer (China) and cameras with a $2.2^{\prime}-3.4^{\prime}$ field of view for $1200-6000-\AA \mathrm{UV}$ and optical imaging (Spain). Its UV-optimised mirror coatings would give it an ultraviolet spectroscopic performance as good as or better than that of the Hubble Space Telescope. Its operational lifetime in geosynchronous orbit would be 5 years, with a 10 -year goal.

\section{Some Conclusions}

- In common with many other astrophysical research fields, planetary nebula studies need multi-wavelength spectroscopic and imaging coverage from space. Planetary nebula researchers therefore need to make a full contribution to future mission definition studies.

- Cancellation of the James Webb Space Telescope could have catastrophic consequences for US space science and technology leadership.

- The decrease in the number of astrophysics space missions per decade, evident from Table 1 , needs to be reversed. This may imply proposing somewhat less ambitious and certainly less costly missions in the future.

- New missions should aim for operational lifetimes of 10-15 years.

- Space agencies appear not to like long-term operational commitments. Astronomers should consider pushing for other bodies to take over responsibility for post-launch operations of future missions, e.g. to take effect circa 3-5 years after launch.

\section{References}

Arnaboldi, M., Freeman, K. C., Mendez, R. H., Capaccioli, M., Ciardullo, R., Ford, H., Gerhard, O.; Hui, X., Jacoby, G. H., Kudritzki, R.-P., \& Quinn, P. J. 1996, ApJ, 472, 145

Bernard-Salas, J., Peeters, E., Sloan, G. C., Cami, J., Guiles, S., \& Houck, J. R. 2006, ApJ, 652, L29

Bernard-Salas, J., Peeters, E., Sloan, G. C., Gutenkunst, S., Matsuura, M., Tielens, A. G. G. M., Zijlstra, A. A., \& Houck, J. R. 2009, ApJ, 652, L29

Groenewegen, M. A. T., et al. 2007, MNRAS, 376, 313

Méndez, R. H., Guerrero, M. A., Freeman, K. C., Arnaboldi, M., Kudritzki, R.-P., Hopp, U., Capaccioli, M., \& Ford, H. 1997, ApJ, 491, L23

Sloan, G. C., Kraemer, K. E., Matsuura, M., Wood, P. R., Price, S. D., \& Egan, M. P. 2006, ApJ, 645, 1118

Swinyard, B. \& Nakagawa, T. 2009, Exp. Astr., 23, 193

Theuns, T. \& Warren, S. J. 1997, MNRAS, 284, L11 